\title{
DENSITY OF INTEGER SEQUENCES
}

\author{
R. KAUFMAN
}

1. Let $N=\left\{n_{k}\right\}$ be an increasing sequence of positive integers. Then $N$ fulfills condition $D$ if it contains a sequence of blocks

$$
B,=\left[u_{\nu}, v_{\nu}\right] \cap N, \quad 1 \leqq u_{\nu}<v_{\nu},
$$

for which $v_{\nu}-u_{\nu} \rightarrow \infty$, and $1+v_{\nu}-u_{\nu} \leqq C \cdot\left|B_{\nu}\right|$. Here $|S|$ always denotes the number of elements in a finite set $S$.

Let $N^{1}, \cdots, N^{r}$ be $r$ sequences fulfilling condition $D$, and for each real number $x$, let

$$
\Delta(x)=\left\{\left(n_{1} x, \cdots, n_{r} x\right): n_{1}<n_{2}<\cdots<n_{r} \text { and } n_{s} \in N^{s}(1 \leqq s \leqq r)\right\} .
$$

Thus $\Delta(x)$ is a denumerable subset of $R^{r}$.

TheOREM 1. For all but a denumerable set of real numbers $x, \Delta(x)$ is dense (modulo $2 \pi)$ in $R^{r}$.

The statement for $r=1$ is proved, with more precision, by Amice [1] and Kahane [2]. It will be clear from the proof that the inequalities in the definition of $\Delta(x)$ can be strengthened almost arbitrarily.

First we express the exceptional set in Theorem 1 as a denumerable union of closed sets. Let $U_{1}, \cdots, U_{j}, \cdots$ be a sequence of open sets in $R^{r}$ forming a base for the topology, and $\Lambda$ the subgroup of $R^{r}$ of integral vectors. Put, for each $j$,

$$
E_{j}=\left\{x \in R: \Delta(x) \cap\left(U_{j}+2 \pi \Lambda\right)=\varnothing\right\} .
$$

Then each $E_{j}$ is closed and we must show that each is denumerable. In the opposite case some $E_{j}$ would contain a compact nondenumerable subset; hence a homeomorph of the Cantor set, and so $E_{j}$ would carry a continuous probability measure $\mu,(\mu(D)=0$ for every denumerable set $D$ ). We shall now state a theorem on probability distributions that implies Theorem 1.

2. Let $M$ be the set of $r$-tuples $\left(n_{1}, \cdots, n_{r}\right) \in N^{1} \times \cdots \times N^{r}$ defined by the inequalities $n_{1}<n_{2}<\cdots<n_{r}$ and $X$ a real random variable whose distribution is continuous.

THEOREM 2. For a certain sequence $\left(n_{i 1}, \cdots, n_{i r}\right) \in M$, the $r$-dimensional variables $Y_{i}=\left(n_{i 1} X, \cdots, n_{i r} X\right), i=1,2,3, \cdots$, tend modulo $2 \pi$ to uniform distribution.

Received by the editors July 25, 1967. 
Deduction of Theorem 1. We suppose, after our remarks on Theorem 1, that for some open set $U$ (one of the $U_{j}$, for example)

$$
P\{\Delta(X) \cap(U+2 \pi \Lambda)=\varnothing\}=1 .
$$

But then $P\left\{Y_{i} \in U+2 \pi \Lambda\right\}=0, i=1,2,3, \cdots$, contradicting the fact that $U+2 \pi \Lambda$ has positive measure in the quotient group $R^{r} / 2 \pi \Lambda$. This completes the deduction of Theorem 1.

3. Proof of Theorem 2. By Weyl's criterion, a sequence $Y_{i}$ tends to uniform distribution (modulo $2 \pi$ ) if the $r$-dimensional characteristic functions, $\psi_{i}$, of the $Y_{i}$ converge to 0 at each element of $\Lambda$ except 0 . For the variables $Y_{i}$, the characteristic functions $\psi_{i}$ are determined by the characteristic function $\phi$ of the real variable $X$ :

$$
\psi_{i}\left(m_{1}, \cdots, m_{r}\right)=\phi\left(m_{1} n_{i 1}+\cdots+m_{r} n_{i r}\right) .
$$

A theorem of Wiener [3, p. 221] states that

$$
\lim _{m \rightarrow \infty} \frac{1}{m+1} \sum_{0}^{m}|\phi(j)|=0,
$$

and in fact the proof cited shows that

$$
\lim _{m \rightarrow \infty} \frac{1}{m+1} \sum_{p}^{p+m}|\phi(j)|=0,
$$

uniformly for all integers $p$.

For any finite set $H$ of elements $\neq 0$ of $\Lambda$, and any $\delta>0$, we shall find a finite subset $S$ of $M$ so that

$$
\sum_{S}\left|\phi\left(m_{1} n_{1}+\cdots+m_{r} n_{r}\right)\right|<\delta|S|
$$

for each $\left(m_{1}, \cdots, m_{r}\right) \in H$. Then, for at least one $\left(n_{1}^{*}, \cdots, n_{r}^{*}\right) \in S$, and for every $\left(m_{1}, \cdots, m_{r}\right) \in H$,

$$
\left|\phi\left(m_{1} n_{1}^{*}+\cdots+m_{r} n_{r}^{*}\right)\right|<\delta|H| ;
$$

this easily implies the existence of the asserted sequence $\left(n_{i 1}, \cdots, n_{i r}\right)$ in $M$. The proof will show that $S$ can be chosen so that (2) holds uniformly for any $\left(m_{1}, \cdots, m_{r}\right)$ containing at least one coefficient $m_{j} \neq 0$ but $\left|m_{j}\right| \leqq C^{\prime}$.

Given a number $b>1$, choose, using (1), intervals $\left[u_{1}, v_{1}\right], \cdots$, $\left[u_{r}, v_{r}\right]$ such that

and

$$
b \leqq v_{1}-u_{1}, \quad b v_{s} \leqq v_{s+1}-u_{s+1}, \quad 1 \leqq s<r,
$$




$$
1+v_{s}-u_{s} \leqq C \cdot\left|N^{s} \cap\left[u_{s}, v_{s}\right]\right|, \quad 1 \leqq s \leqq r .
$$

Hence

$$
\prod_{s=1}^{r}\left(v_{s}-u_{s}+1\right) \leqq C^{r} \prod_{s=1}^{r}\left|N^{s} \cap\left[u_{s}, v_{s}\right]\right|
$$

Let $Q$ denote the rectangle in $R^{r}\left[u_{1}, v_{1}\right] \times \cdots \times\left[u_{r}, v_{r}\right]$, so that the last inequality is just

$$
|Q \cap \Lambda|=\prod_{s=1}^{r}\left(v_{s}-u_{s}+1\right) \leqq C^{r}\left|Q \cap N^{1} \times \cdots \times N^{r}\right| .
$$

Moreover, the inequalities $n_{1}<\cdots<n_{r}$ are satisfied by all the elements of $Q \cap \Lambda$ except at most $r b^{-1}|Q \cap \Lambda|$. Thus if $b$ is sufficiently large

$$
|Q \cap \Lambda| \leqq 2 C^{r}|Q \cap M|
$$

It is enough to attain, therefore,

$$
\sum_{Q \cap \Lambda}\left|\phi\left(m_{1} n_{1}+\cdots+m_{r} n_{r}\right)\right| \leqq \frac{1}{2} \delta C^{-r}|Q \cap \Lambda| .
$$

Suppose, for example, that $1 \leqq m_{r} \leqq C^{\prime}$. By holding $n_{1}, \cdots, n_{r-1}$ fixed, and varying $n_{r}$ in $\left[u_{r}, v_{r}\right]$, we obtain from the sums $m_{1} n_{1}+\cdots$ $+m_{r} n_{r}$ an arithmetic progression of at least $b$ terms, and difference at most $C^{\prime}$. So

$$
\sum_{u_{r} \leqq n_{r} \leqq v_{r}}\left|\phi\left(m_{1} n_{1}+\cdots+m_{r} n_{r}\right)\right| \leqq \sup _{p} \sum_{p}^{p+h}|\phi(j)|,
$$

where $b \leqq v_{r}-u_{r+1} \leqq h \leqq C^{\prime}\left(v_{r}-u_{r}+1\right)$. Hence

$$
\sup _{p} \sum_{p+1}^{p+h}|\phi(j)|=o(h)=o\left(v_{r}-u_{r}+1\right) \quad \text { as } b \rightarrow \infty .
$$

These estimates are uniform with respect to $n_{1}, \cdots, n_{r-1}$ and so (3) holds if $b$ is sufficiently large. Theorem 2 is completely proved.

\section{REFERENCES}

1. Y. Amice, Un théorème de finitude, Ann. Inst. Fourier 14 (1964), 527-531.

2. J.-P. Kahane, Sur les mauvaises répartitions modulo 1, Ann. Inst. Fourier 14 (1964), 519-526.

3. A. Zygmund, Trigonometric series, Monogr. Mat., Bd. 5, PWN, Warsaw, 1935.

UNIVERSITY OF ILLINOIS 